\title{
Parameterized Model Order Reduction Using Extended Balanced Truncation*
}

\author{
Henrik Sandberg ${ }^{1}$
}

\begin{abstract}
We show how extended balanced truncation can be applied to the parameterized model order reduction (PMOR) problem. Two variants are introduced and are shown to improve already available $a$ priori error bounds, as well as actual approximation errors in a numerical experiment.
\end{abstract}

\section{INTRODUCTION}

Model (order) reduction concerns systematic approximation of complex models. The topic has received a great deal of attention in the control community at least since the early 1980's, and the books [1], [2] give good surveys of the field. Model reduction is also of prime importance in other communities, such as in fluid mechanics and circuit design. For many application areas it is of interest to reduce parameter-dependent models, which gives rise to the so-called parameterized model order reduction (PMOR) problem, see [3], [4] and references therein. In a PMOR problem, a parameter-varying model is to be approximated by a similarly parameter-varying reduced-order model. The parameters can represent different operating conditions or uncertainties at the time of approximation, for example. In the control community, a related problem is model reduction of linear parameter-varying (LPV) and uncertain systems, see for example [5]-[8]. A difference between PMOR and model reduction of LPV systems is that in the latter case the parameters are often time varying and the models necessarily take a linear state-space form. In this paper, we will consider linear state-space systems which are affine in non-timevarying parameters. Hence the treated problem is more restrictive than model reduction of general LPV systems, and more related to PMOR. By focusing on this class of systems, we can apply extended balanced truncation [9] and will be able to devise two new methods which improve both approximation errors and a priori error bounds, as compared to direct application of the methods in [5]-[8]. However, it should be noted that the methods in [5]-[8] can handle more general systems, and the advantage of the two new methods lies in their ability to better exploit the given model structure. The methods developed in [3], [4] are more suitable for approximating parameterized models of very high order. The methods presented here can only solve problems of moderate order. On the other hand, they come with the already mentioned error bounds and with

*This work was supported in part by the Swedish Research Council under Grants 2007-6350 and 2009-4565, and the Swedish Foundation for Strategic Research under the ICT- $\Psi$ project.

${ }^{1} \mathrm{H}$. Sandberg is with the KTH Royal Institute of Technology, ACCESS Linnaeus Centre, School of Electrical Engineering, Automatic Control Lab, Stockholm, Sweden hsan@kth. se
Hankel-like singular values which can be used to determine suitable approximation order, which is not available in [3], [4]. Hence, it appears the introduced methods are a valuable addition and bring new insight to the PMOR problem.

The main contribution of this paper is that we apply the theory of extended balanced truncation [9] to a PMOR problem. In [9], it was shown that by introducing extra slack variables in generalized balanced truncation (see [6], [10]) it is possible to improve the balanced truncation $\mathcal{H}_{\infty}$ norm error bound, and to enforce topological constraints in the reduced model. Here we note that the same slack variables can be used to strengthen available error bounds and approximation errors in PMOR. The idea of introducing extra slack variables in linear matrix inequalities (LMIs) was first presented in [11]. The original motivation for doing so was to compute Lyapunov functions for analysis and controller synthesis for parameter-varying systems, see [11] [13]. Here we apply similar ideas to the PMOR problem.

The organization of the paper is as follows. In Section II, we introduce the parameter-varying systems. In Section III, different types of parameter-varying generalized Gramians are introduced, and convex optimization problems are proposed for their computation. In Section IV, we show how the parameter-varying Gramians can be used to obtain two new methods for model reduction, and study an example.

\section{Parameter-Varying Linear Systems}

The parameter-varying linear systems we consider are in the form

$$
G_{\xi}\left\{\begin{aligned}
x(k+1) & =A_{\xi} x(k)+B_{\xi} u(k) \\
y(k) & =C_{\xi} x(k)+D_{\xi} u(k)
\end{aligned}\right.
$$

where $x(k) \in \mathbb{R}^{n}, u(k) \in \mathbb{R}^{m}, y(k) \in \mathbb{R}^{p}$, and $k \in \mathbb{Z}$ is the discrete-time variable. The admissible realizations belong to a convex bounded polyhedron given by

$$
\begin{aligned}
M_{\xi} & =\left[\begin{array}{ll}
A_{\xi} & B_{\xi} \\
C_{\xi} & D_{\xi}
\end{array}\right]:=\sum_{i=1}^{N} \xi_{i}\left[\begin{array}{cc}
A_{i} & B_{i} \\
C_{i} & D_{i}
\end{array}\right], \quad \xi \in \Xi \\
A_{i} & \in \mathbb{R}^{n \times n}, \quad B_{i} \in \mathbb{R}^{n \times m}, \quad C_{i} \in \mathbb{R}^{p \times n}, \quad D_{i} \in \mathbb{R}^{p \times m} \\
\Xi & :=\left\{\xi: \xi \in \mathbb{R}^{N}, \sum_{i=1}^{N} \xi_{i}=1, \xi_{i} \geq 0, i=1, \ldots, N\right\} .
\end{aligned}
$$

The parameter $\xi$ is assumed fixed in time, and for each $\xi$, $G_{\xi}$ has the transfer function

$$
G_{\xi}(z)=\mathcal{F}_{u}\left(M_{\xi}, z^{-1} I_{n}\right):=C_{\xi}\left(z I-A_{\xi}\right)^{-1} B_{\xi}+D_{\xi}
$$


where $z \in \mathbb{C}$. With $\|u\|_{\left[k_{1}, k_{2}\right]}$ we mean the 2-norm $\|u\|_{\left[k_{1}, k_{2}\right]}:=\left(\sum_{k=k_{1}}^{k_{2}} u(k)^{\top} u(k)\right)^{1 / 2}$. The induced 2-norm of $G_{\xi}$ over the time interval $[0, \infty]$ is equal to the $\mathcal{H}_{\infty}$-norm of $G_{\xi}(z),\left\|G_{\xi}\right\|_{\infty}:=\sup _{z \in \mathbb{C} \backslash \overline{\mathbb{D}}} \bar{\sigma}\left(G_{\xi}(z)\right)$, see [14]. Here $\overline{\mathbb{D}}$ is the closed unit disc in the complex plane $\mathbb{C}$. For inputoutput stable systems $G_{\xi}$ the norm $\left\|G_{\xi}\right\|_{\infty}$ is finite.

A seemingly direct approach to approximate (1) would be to solve the Lyapunov equations

$$
\begin{aligned}
& P(\xi)-A_{\xi} P(\xi) A_{\xi}^{\top}-B_{\xi} B_{\xi}^{\top}=0 \\
& Q(\xi)-A_{\xi}^{\top} Q(\xi) A_{\xi}-C_{\xi}^{\top} C_{\xi}=0
\end{aligned}
$$

for the parameter-varying Gramians $P(\xi)$ and $Q(\xi)$ (assuming $\rho\left(A_{\xi}\right)<1$ ), and then proceed by applying balanced truncation or Hankel-norm approximation (see [1], [2]). However, it is generally not possible to express the Gramians $P(\xi)$ and $Q(\xi)$ as simple functions of $\xi$, and the same thus holds for the resulting reduced-order model $\hat{G}(z, \xi)$. Hence, to apply this approach one would need to solve for the Gramians numerically for every choice of $\xi$, which can be very time consuming, or even impossible, if many different parameter choices $\xi \in \Xi$ are to be used.

In this paper, we will not pursue this direction but instead explore methods that generate generalized Gramians [6], [10] and extended Gramians [9] with a simpler, constant or affine, parameter dependence. Then we show how (when possible) to obtain $q$-th-order reduced models $\hat{G}_{\xi}(q<n)$ in the form

$$
\begin{aligned}
\hat{M}_{\xi} & =\left[\begin{array}{ll}
\hat{A}_{\xi} & \hat{B}_{\xi} \\
\hat{C}_{\xi} & \hat{D}_{\xi}
\end{array}\right]=\sum_{i=1}^{N} \xi_{i}\left[\begin{array}{cc}
\hat{A}_{i} & \hat{B}_{i} \\
\hat{C}_{i} & \hat{D}_{i}
\end{array}\right], \quad \xi \in \Xi \\
\hat{A}_{i} & \in \mathbb{R}^{q \times q}, \quad \hat{B}_{i} \in \mathbb{R}^{q \times m}, \quad \hat{C}_{i} \in \mathbb{R}^{p \times q}, \quad \hat{D}_{i} \in \mathbb{R}^{p \times m} \\
\hat{G}_{\xi}(z) & =\mathcal{F}_{u}\left(\hat{M}_{\xi}, z^{-1} I_{q}\right), \quad z \in \mathbb{C},
\end{aligned}
$$

such that $\left\|G_{\xi}-\hat{G}_{\xi}\right\|_{\infty}$ is guaranteed to be small for all $\xi \in \Xi$.

Remark 1: By $\bullet_{\xi}$ we mean that $\bullet$ can be expressed as a convex combination, $\bullet_{\xi}:=\sum_{i=1}^{N} \xi_{i} \bullet, \xi \in \Xi$. If we use the notation $\bullet(\xi)$, we mean that $\bullet$ has a more complicated dependence on the parameter $\xi$.

\section{PARAMETER-VARYING GRAMIANS}

It is well known that generalized balanced truncation and optimal $\mathcal{H}_{\infty}$-norm model reduction use generalized Gramians $P$ and $Q$ satisfying Lyapunov inequalities

$$
\begin{array}{ll}
P(\xi)-A_{\xi} P(\xi) A_{\xi}^{\top}-B_{\xi} B_{\xi}^{\top}>0, & P(\xi)>0 \\
Q(\xi)-A_{\xi}^{\top} Q(\xi) A_{\xi}-C_{\xi}^{\top} C_{\xi}>0, & Q(\xi)>0
\end{array}
$$

where $P(\xi) \in \mathbb{R}^{n \times n}$ and $Q(\xi) \in \mathbb{R}^{n \times n}$ are assumed to be symmetric (see [10]). These LMIs are are solvable iff $\rho\left(A_{\xi}\right)<1$. Before discussing how to solve (3)-(4), we note that the LMIs can be extended by introducing extra slack variables $R$ and $S$, see [11]. The corresponding extended
Lyapunov inequalities are given by

$$
\begin{gathered}
{\left[\begin{array}{ccc}
P(\xi) & A_{\xi} R(\xi) & B_{\xi} \\
R(\xi)^{\top} A_{\xi}^{\top} & R(\xi)+R(\xi)^{\top}-P(\xi) & 0 \\
B_{\xi}^{\top} & 0 & I
\end{array}\right]>0,} \\
{\left[\begin{array}{ccc}
S(\xi)+S(\xi)^{\top}-Q(\xi) & S(\xi) A_{\xi} & 0 \\
A_{\xi}^{\top} S(\xi)^{\top} & Q(\xi) & C_{\xi}^{\top} \\
0 & C_{\xi} & I
\end{array}\right]>0,}
\end{gathered}
$$

with extended controllability Gramian $(P, R)$ and extended observability Gramian $(Q, S)$ [9]. Note that $R(\xi) \in \mathbb{R}^{n \times n}$ and $S(\xi) \in \mathbb{R}^{n \times n}$ are not necessarily symmetric matrices.

The following proposition is a reformulation of a result in [9], and is an application of ideas first presented in [11]. The proposition motivates why we call $R$ and $S$ slack variables.

Proposition 1: (Based on [9], [11]) Suppose $\xi \in \mathbb{R}^{N}$ is fixed, $P(\xi), Q(\xi) \in \mathbb{R}^{n \times n}$ are symmetric, and $R(\xi), S(\xi) \in$ $\mathbb{R}^{n \times n}$. Then the following holds:

(i) If $P(\xi)$ satisfies (3), then there exists an $R(\xi)$ such that $P(\xi)$ and $R(\xi)$ satisfy (5). Conversely, if $P(\xi)$ and $R(\xi)$ satisfy (5), then $P(\xi)$ satisfies (3).

(ii) If $Q(\xi)$ satisfies (4), then there exists an $S(\xi)$ such that $Q(\xi)$ and $S(\xi)$ satisfy (6). Conversely, if $Q(\xi)$ and $S(\xi)$ satisfy (6), then $Q(\xi)$ satisfies (4).

In [9], the extended Gramians were used to improve error bounds for balanced truncation. Here, we will instead exploit that there are no products of the type $A_{\xi} P(\xi)$ or $A_{\xi}^{\top} Q(\xi)$ in (5) and (6). This observation has been used for parametervarying controller synthesis in, for example, [12], [13].

Next, two methods for computing generalized Gramians $P(\xi)$ and $Q(\xi)$, valid for all $\xi \in \Xi$, are presented.

\section{A. Computing Constant Generalized Gramians}

The first simple idea is to search for constant generalized Gramians

$$
P(\xi)=P, \quad Q(\xi)=Q,
$$

that satisfy (3) and (4) for all $\xi \in \Xi$. If we assume the system $G_{\xi}$ is quadratically stable [11], such Gramians exist. In fact, it can be verified using Schur complements and linearity that it is enough to satisfy the LMIs at the corners of the convex polyhedron $M_{\xi}$. Thus we propose the following semidefinite programs to be solved.

$$
\begin{array}{cl}
\underset{P}{\operatorname{minimize}} & \operatorname{trace}(P) \\
\text { subject to } & P-A_{i} P A_{i}^{\top}-B_{i} B_{i}^{\top}>0 \\
& P>0, \quad i=1, \ldots, N \\
\underset{Q}{\operatorname{minimize}} & \operatorname{trace}(Q) \\
\text { subject to } & Q-A_{i}^{\top} Q A_{i}-C_{i}^{\top} C_{i}>0 \\
& Q>0, \quad i=1, \ldots, N .
\end{array}
$$

We denote the optimal solutions (since they exist by the assumption of quadratic stability) by $P^{\star}$ and $Q^{\star}$. The reason we minimize the traces of the Gramians is to obtain unique solutions, and because "small" generalized Gramians are more useful. But one could consider to minimize other convex objective functions as well. 
Remark 2: To use constant generalized Gramians to reduce LPV and uncertain systems has certainly been used before, see, for example, [5], [6], [8]. Here we include this method for comparison.

\section{B. Computing Affine Generalized Gramians}

We now relax the assumption (7) and search for affine generalized Gramians in the form

$$
P(\xi)=P_{\xi}=\sum_{i=1}^{N} \xi_{i} P_{i}, \quad Q(\xi)=Q_{\xi}=\sum_{i=1}^{N} \xi_{i} Q_{i},
$$

for all $\xi \in \Xi$. Directly inserting these relations into (3) and (4) unfortunately leads to non-convex problems due to the products $A_{\xi} P_{\xi}$ and $A_{\xi}^{\top} Q_{\xi}$. One could try to solve these non-convex problems using various relaxation techniques, for example those found in [15] or [16] ${ }^{1}$. The idea of this paper is instead to use (5)-(6), and Proposition 1. In order to obtain a convex problem, we impose that the slack variables should be constant,

$$
R(\xi)=R, \quad S(\xi)=S, \quad \forall \xi \in \Xi .
$$

As before, due to linearity, it is enough to solve the (extended) LMIs in the corners of $M_{\xi}$. We propose the following semidefinite programs:

$$
\begin{array}{clc}
\underset{P_{i}, R}{\operatorname{minimize}} & \sum_{i=1}^{N} \operatorname{trace}\left(P_{i}\right) \\
\text { subject to } & {\left[\begin{array}{ccc}
P_{i} & A_{i} R & B_{i} \\
R^{\top} A_{i}^{\top} & R+R^{\top}-P_{i} & 0 \\
B_{i}^{\top} & 0 & I
\end{array}\right]>0} \\
& P_{i} \leq P^{\star}, \quad i=1, \ldots, N \\
\underset{Q_{i}, S}{\operatorname{minimize}} & \sum_{i=1}^{N} \operatorname{trace}\left(Q_{i}\right) \\
\text { subject to } & {\left[\begin{array}{ccc}
S+S-Q_{i} & S A_{i} & 0 \\
A_{i}^{\top} S^{\top} & Q_{i} & C_{i}^{\top} \\
0 & C_{i} & I
\end{array}\right]>0}
\end{array}
$$

From the optimal solutions $P_{i}^{\star}$ and $Q_{i}^{\star}$ we construct affine Gramians, denoted by

$$
P_{\xi}^{\star}:=\sum_{i=1}^{N} \xi_{i} P_{i}^{\star}, \quad Q_{\xi}^{\star}:=\sum_{i=1}^{N} \xi_{i} Q_{i}^{\star} .
$$

It follows from (12)-(13) that $P_{\xi}^{\star}>0$ and $Q_{\xi}^{\star}>0$ for all $\xi \in \Xi$. Note also that by Proposition 1, the affine Gramians $P_{\xi}^{\star}$ and $Q_{\xi}^{\star}$ satisfy (3)-(4). The affine Gramians are "better" than the constant Gramians in Section III-A in the sense that

$$
P_{\xi}^{\star} \leq P^{\star}, \quad Q_{\xi}^{\star} \leq Q^{\star}, \quad \forall \xi \in \Xi,
$$

due to the constraints $P_{i} \leq P^{\star}, Q_{i} \leq Q^{\star}$, and (14). In fact, these constraints are only included to ensure the affine Gramians are better when $G_{\xi}$ is quadratically stable [11].

\footnotetext{
${ }^{1}$ The author thanks an anonymous reviewer for pointing this out.
}

If the system is not quadratically stable, the LMIs (12) and (13) may still be feasible if $P_{i} \leq P^{\star}$ and $Q_{i} \leq Q^{\star}$ are dropped. Hence, extended Gramians may exist and could be used to reduce systems that are not quadratically stable. We have here chosen to minimize the sum of the traces of $P_{i}$ and $Q_{i}$ to ensure the Gramians are small everywhere. One could consider other convex objective functions and constraints which would force the Gramians to be uniformly small. This is an interesting topic for future research.

One reason small generalized and extended Gramians are desirable is because they provide tighter bounds on observability and reachability as formalized in the following proposition.

Proposition 2: Suppose $P^{\star}, Q^{\star}, P_{\xi}^{\star}$, and $Q_{\xi}^{\star}$ as defined above exist, and that the system $G_{\xi}$, for a fixed $\xi \in \Xi$, is released from the state $x(0)$. Then the following holds for $0 \leq k \leq \infty$ :

(i) If the input is $u(i)=0,0 \leq i \leq k$, the output signal energy is bounded as

$$
\begin{aligned}
x(k+1)^{\top} Q_{\xi}^{\star} x(k+1)+\|y\|_{[0, k]}^{2} & \leq x(0)^{\top} Q_{\xi}^{\star} x(0) \\
& \leq x(0)^{\top} Q^{\star} x(0) .
\end{aligned}
$$

(ii) For arbitrary inputs $u(k)$, the set of reachable states $x(k)$ are bounded as

$$
\begin{gathered}
x(k+1)^{\top}\left(P^{\star}\right)^{-1} x(k+1) \leq x(k+1)^{\top}\left(P_{\xi}^{\star}\right)^{-1} x(k+1) \\
\leq\|u\|_{[0, k]}^{2}+x(0)^{\top}\left(P_{\xi}^{\star}\right)^{-1} x(0) .
\end{gathered}
$$

\section{Computing Extended Gramians}

In Section III-B the extended LMIs (5)-(6) were used to compute affine generalized Gramians. But in [9] it was shown that the slack variables $R$ and $S$ themselves can be used to reduce models. Therefore, we propose the following semidefinite programs, which are slightly modified versions of (12) and (13).

$$
\begin{aligned}
& \underset{P_{i}, R}{\operatorname{minimize}} \operatorname{trace}(R) \\
& \text { subject to }\left[\begin{array}{ccc}
P_{i} & A_{i} R & B_{i} \\
R^{\top} A_{i}^{\top} & R+R^{\top}-P_{i} & 0 \\
B_{i}^{\top} & 0 & I
\end{array}\right]>0 \\
& R=R^{\top} \leq P^{\star}, \quad i=1, \ldots, N \\
& \underset{Q_{i}, S}{\operatorname{minimize}} \operatorname{trace}(S) \\
& \text { subject to }\left[\begin{array}{ccc}
S+S-Q_{i} & S A_{i} & 0 \\
A_{i}^{\top} S^{\top} & Q_{i} & C_{i}^{\top} \\
0 & C_{i} & I
\end{array}\right]>0 \\
& S=S^{\top} \leq Q^{\star}, \quad i=1, \ldots, N .
\end{aligned}
$$

We denote the optimal solutions by $R^{\star}$ and $S^{\star}$ (which exist if (12)-(13) are feasible), and note that $R^{\star} \leq P^{\star}$ and $S^{\star} \leq Q^{\star}$.

\section{Parameterized Model Order Reduction}

Three sets of Gramian(-like) matrices have been introduced: $\left(P^{\star}, Q^{\star}\right),\left(P_{\xi}^{\star}, S_{\xi}^{\star}\right)$, and $\left(R^{\star}, S^{\star}\right)$. Each set gives rise to a parameterized model-reduction procedure with a priori 
error bounds. This will be explained next, and a comparison on an example is presented in Section IV-B.

Let us consider constant state coordinate transformations $\bar{x}=T x$ of $G_{\xi}$, where $T$ is invertible. One can show [9] that under such transformations, the solutions to (8)-(9) and (16)-(17) transform as

$$
\begin{aligned}
\bar{P}^{\star} & =T P^{\star} T^{\top}, & \bar{R}^{\star} & =T R^{\star} T^{\top}, \\
\bar{Q}^{\star} & =T^{-\top} Q^{\star} T^{-1}, & \bar{S}^{\star} & =T^{-\top} S^{\star} T^{-1} .
\end{aligned}
$$

For the affine Gramians $P_{\xi}^{\star}$ and $Q_{\xi}^{\star}$ it is more natural to consider parameterized transformations $\bar{x}=T(\xi) x$ and then

$$
\bar{P}(\xi)^{\star}=T(\xi) P_{\xi}^{\star} T(\xi)^{\top}, \quad \bar{Q}(\xi)^{\star}=T(\xi)^{-\top} Q_{\xi}^{\star} T(\xi)^{-1} .
$$

The eigenvalues of the Gramian products are coordinate independent as stated next.

Lemma 1: The eigenvalues

$$
\begin{aligned}
\sigma_{i} & :=\sqrt{\lambda_{i}\left(P^{\star} Q^{\star}\right)}, \quad \sigma_{i}(\xi):=\sqrt{\lambda_{i}\left(P_{\xi}^{\star} S_{\xi}^{\star}\right)} \\
\sigma_{e, i} & :=\sqrt{\lambda_{i}\left(R^{\star} S^{\star}\right)}, \quad i=1, \ldots, n,
\end{aligned}
$$

are non-negative real numbers (each sorted in decreasing order), and are invariant under transformations $\bar{x}=T x$.

Note that the eigenvalues $\sigma_{i}(\xi)$ (along with $\bar{P}(\xi)^{\star}$ and $\left.\bar{Q}(\xi)^{\star}\right)$ are generally not affine in $\xi$, as indicated by the notation (see Remark 1). Furthermore, the eigenvalues have the following property.

Theorem 1: The eigenvalues in Lemma 1 satisfy the bounds $\sigma_{e, i} \leq \sigma_{i}$ and $\sigma_{i}(\xi) \leq \sigma_{i}$, for $i=1, \ldots, n$ and all $\xi \in \Xi$.

Proof: The eigenvalues $\sigma_{e, 1}$ and $\sigma_{1}$ can be expressed as generalized Rayleigh quotients,

$$
\begin{aligned}
\sigma_{e, 1}=\lambda_{1}\left(R^{\star} S^{\star}\right) & =\max _{x} \frac{x^{\top} S^{\star} x}{x^{\top}\left(R^{\star}\right)^{-1} x} \\
& \leq \max _{x} \frac{x^{\top} Q^{\star} x}{x^{\top}\left(P^{\star}\right)^{-1} x}=\sigma_{1},
\end{aligned}
$$

since $R^{\star} \leq P^{\star}$ and $S^{\star} \leq Q^{\star}$. Corresponding bounds can be established for $\sigma_{e, 2}, \sigma_{2}, \ldots$, and for $\sigma_{i}(\xi)$ using the CourantFischer min-max principle.

Just as in regular balanced truncation, see for example [14], we can find transformations to balance the Gramians. In these coordinates the eigenvalues end up on the diagonals,

$$
\begin{aligned}
T_{1} P^{\star} T_{1}^{\top} & =T_{1}^{-\top} Q^{\star} T_{1}^{-1} \\
& =\Sigma:=\operatorname{diag}\left\{\sigma_{1}, \ldots, \sigma_{n}\right\} \\
T_{2} R^{\star} T_{2}^{\top} & =T_{2}^{-\top} S^{\star} T_{2}^{-1} \\
& =\Sigma_{e}:=\operatorname{diag}\left\{\sigma_{e, 1}, \ldots, \sigma_{e, n}\right\} \\
T_{3}(\xi) P_{\xi}^{\star} T_{3}(\xi)^{\top} & =T_{3}(\xi)^{-\top} Q_{\xi}^{\star} T_{3}(\xi)^{-1} \\
& =\Sigma(\xi):=\operatorname{diag}\left\{\sigma_{1}(\xi), \ldots, \sigma_{n}(\xi)\right\} .
\end{aligned}
$$

Let us first apply balanced truncation using $\left(P^{\star}, Q^{\star}\right)$ and $\left(R^{\star}, S^{\star}\right)$ since this is done with constant transformations $T_{1}$ and $T_{2}$, respectively. The realization of $G_{\xi}$ under the coordinate transformations $T_{j}(j=1,2)$ becomes

$$
\bar{M}_{\xi, j}=\left[\begin{array}{cc}
\bar{A}_{\xi, j} & \bar{B}_{\xi, j} \\
\bar{C}_{\xi, j} & D_{\xi}
\end{array}\right]:=\sum_{i=1}^{N} \xi_{i}\left[\begin{array}{cc}
T_{j}^{-1} A_{i} T_{j} & T_{j}^{-1} B_{i} \\
C_{i} T_{j} & D_{i}
\end{array}\right] .
$$

Also introduce the partition

$$
\begin{aligned}
& \bar{A}_{\xi, j}=\left[\begin{array}{cc}
\bar{A}_{\xi, j}^{11} & \bar{A}_{\xi, j}^{12} \\
\bar{A}_{\xi, j}^{21} & \bar{A}_{\xi, j}^{22}
\end{array}\right], \bar{B}_{\xi, j}=\left[\begin{array}{c}
\bar{B}_{\xi, j}^{1} \\
\bar{B}_{\xi}^{2, j}
\end{array}\right], \bar{C}_{\xi, j}=\left[\begin{array}{c}
\bar{C}_{\xi, j}^{1 \top} \\
\bar{C}_{\xi, j}^{2 \dagger}
\end{array}\right]^{\top}, \\
& \bar{A}_{\xi, j}^{11} \in \mathbb{R}^{q \times q}, \quad \bar{B}_{\xi, j}^{1} \in \mathbb{R}^{q \times m}, \quad \bar{C}_{\xi, j}^{1} \in \mathbb{R}^{p \times q},
\end{aligned}
$$

where $q<n$ will correspond to the order of the reduced model. We can truncate the lower/right block elements of the realization $\bar{M}_{\xi, j}$ to obtain a $q$-th-order realization

$$
\hat{M}_{\xi, j}=\left[\begin{array}{cc}
\bar{A}_{\xi, j}^{11} & \bar{B}_{\xi, j}^{1} \\
\bar{C}_{\xi, j}^{1} & D_{\xi}
\end{array}\right]=\sum_{i=1}^{N} \xi_{i}\left[\begin{array}{cc}
\bar{A}_{i, j}^{11} & \bar{B}_{i, j}^{1} \\
\bar{C}_{i, j}^{1} & D_{i}
\end{array}\right] .
$$

Note that the realization of the resulting reduced-order model

$$
\begin{aligned}
\hat{G}_{\xi, j}(z) & =\mathcal{F}_{u}\left(\hat{M}_{\xi, j}, z^{-1} I_{q}\right) \\
& =\bar{C}_{\xi, j}^{1}\left(z I-\bar{A}_{\xi, j}^{11}\right)^{-1} \bar{B}_{\xi, j}^{1}+D_{\xi}, \quad j=1,2,
\end{aligned}
$$

is still affine in the parameters $\xi$, just as for the original model $G_{\xi}$. Hence, it is easy to compute $\hat{M}_{\xi, j}$ offline, and to construct the desired model $\hat{G}_{\xi, j}$ whenever the actual parameter $\xi$ is known or changes.

The reduced-order model resulting from $\left(P_{\xi}^{\star}, S_{\xi}^{\star}\right)$ is more complicated due to the balancing transformation $T_{3}(\xi)$, which is parameter varying. The balanced realization becomes

$$
\bar{M}_{3}(\xi)=\sum_{i=1}^{N} \xi_{i}\left[\begin{array}{cc}
T_{3}(\xi)^{-1} A_{i} T_{3}(\xi) & T_{3}(\xi)^{-1} B_{i} \\
C_{i} T_{3}(\xi) & D_{i}
\end{array}\right] .
$$

and its truncated version

$$
\hat{M}_{3}(\xi)=\left[\begin{array}{cc}
\bar{A}_{3}^{11}(\xi) & \bar{B}_{3}^{1}(\xi) \\
\bar{C}_{3}^{1}(\xi) & D_{\xi}
\end{array}\right] \in \mathbb{R}^{(q+p) \times(q+m)},
$$

is generally no longer affine in $\xi$. The reduced-order model becomes

$$
\begin{aligned}
\hat{G}_{3}(z, \xi) & =\mathcal{F}_{u}\left(\hat{M}_{3}(\xi), z^{-1} I_{q}\right) \\
& =\bar{C}_{3}^{1}(\xi)\left(z I-\bar{A}_{3}^{11}(\xi)\right)^{-1} \bar{B}_{3}^{1}(\xi)+D_{\xi} .
\end{aligned}
$$

For every new $\xi$, one needs to compute $T_{3}(\xi)$ from $P_{\xi}^{\star}$ and $Q_{\xi}^{\star}$, then construct $\bar{M}_{3}(\xi)$ and truncate it, which is significantly more computationally expensive than constructing $\hat{G}_{\xi, 1}$ and $\hat{G}_{\xi, 2}$. On the other hand, the coordinates in $\hat{G}_{3}(\xi)$ depend on $\xi$ and one may expect better approximations. That this often indeed is the case is shown in the example.

\section{A. A Priori Error Bounds}

Three different reduced-order models have been proposed. The following theorem shows that all of them are endowed with a priori error bounds. Also, the error bounds procured from the extended LMIs (3)-(4) give better bounds.

Theorem 2: The reduced-order parameter-varying systems defined above satisfy the a priori error bounds

$$
\begin{gathered}
\left\|G_{\xi}-\hat{G}_{\xi, 1}\right\|_{\infty} \leq 2 \sum_{i=q+1}^{n} \sigma_{i}=: \delta_{1}(q) \\
\left\|G_{\xi}-\hat{G}_{\xi, 2}\right\|_{\infty} \leq 2 \sum_{i=q+1}^{n} \sigma_{e, i}=: \delta_{2}(q) \\
\left\|G_{\xi}-\hat{G}_{3}(\xi)\right\|_{\infty} \leq 2 \sum_{i=q+1}^{n} \sigma_{i}(\xi)=: \delta_{3}(q, \xi) .
\end{gathered}
$$


Furthermore, it holds that $\delta_{2}(q) \leq \delta_{1}(q)$ and $\delta_{3}(q, \xi) \leq$ $\delta_{1}(q)$, for all $\xi \in \Xi$ and $q=0, \ldots, n-1$.

Proof: The error bounds for $\hat{G}_{\xi, 1}$ and $\hat{G}_{3}(\xi)$ are relatively standard, because these models are obtained from balancing generalized Gramians. Hence, for each $\xi$, we can apply the bound obtained in for example [6] or [10]. That $\delta_{3}(q, \xi)$ is smaller than $\delta_{1}(q)$ follows immediately from Theorem 1.

The bound on $\hat{G}_{\xi, 2}$ follows from an application of a result in [9]: Note that the constant slack variables $R^{\star}$ and $S^{\star}$ by construction satisfy the extended Lyapunov inequalities (5) and (6) for all $\xi \in \Xi$. Hence, for all fixed $\xi \in \Xi$ we can apply the error bound [9, Theorem 2] using $R^{\star}$ and $S^{\star}$, and after balancing and truncation the bound follows. That $\delta_{2}(q)$ is smaller than $\delta_{1}(q)$ follows immediately from Theorem 1.

The error bounds for the reduced models based on the extended LMIs $\left(\hat{G}_{\xi, 2}, \hat{G}_{3}(\xi)\right)$ are smaller than the bound for the more traditional reduced model $\hat{G}_{\xi, 1}$, hence proving their worth. However, in particular choices of $\xi$, it may happen that the actual approximation error is smaller for $\hat{G}_{\xi, 1}$. (This appears to be rare though.) Note also that the error bounds $\delta_{1}(q)$ and $\delta_{2}(q)$ are uniform in $\xi$, whereas $\delta_{3}(q, \xi)$ varies with $\xi$. Thus it may make sense to vary the model order $q$ based on the bound in the latter case. Finally, one may wonder if $\delta_{3}(q, \xi) \leq \delta_{2}(q)$ (or vice versa). This generally does not hold, as seen next.

\section{B. Example}

Consider the parameter-varying linear system $G_{\xi}$ with realization

$$
\begin{aligned}
& A(\alpha)=\left(\begin{array}{cccc}
0.8 & -0.25 & 0 & 1 \\
1 & 0 & 0 & 0 \\
0.8 \alpha & -0.5 \alpha & 0.2 & 0.03+\alpha \\
0 & 0 & 1 & 0
\end{array}\right), \quad|\alpha|<\gamma \\
& B(\beta)=\beta\left(\begin{array}{l}
0 \\
0 \\
1 \\
0
\end{array}\right)+(1-\beta)\left(\begin{array}{l}
1 \\
0 \\
0 \\
0
\end{array}\right), \quad 0 \leq \beta \leq 1 \\
& C=\left(\begin{array}{llll}
1 & 0 & 0 & 0
\end{array}\right), \quad D=0
\end{aligned}
$$

where $A(\alpha)$ and $B(\beta)$ are taken from [11]. It is shown in [11] that $G_{\xi}$ is quadratically stable for $|\alpha|<\gamma=0.4279$ (and stable for $\gamma=0.4619$ ). In the following, we will use $|\alpha| \leq 0.4$ or $|\alpha| \leq 0.04$. In the first case, we are thus close to the limit of being quadratically stable, and the four corners of the convex polyhedron $M_{\xi}$ are $M_{1}=\left[\begin{array}{cc}A(-0.4) & B(0) \\ C & 0\end{array}\right]$, $M_{2}=\left[\begin{array}{cc}A(-0.4) & B(1) \\ C & 0\end{array}\right], M_{3}=\left[\begin{array}{cc}A(0.4) & B(0) \\ C & 0\end{array}\right]$, and $M_{4}=$ $\left[\begin{array}{cc}A(0.4) & B(1) \\ C & 0\end{array}\right]$. We next reduce the order from four $(n=4)$ to two $(q=2)$ by applying the three proposed reduction methods, and also apply normal balanced truncation for some values of $\xi$ for comparison. To solve the involved semidefinite programs, SeDuMi [17] and YALMIP [18] are used.

In Fig. 1, the actual approximation errors are shown along two parameter paths in the set $M_{\xi}$. In Path 1 (which

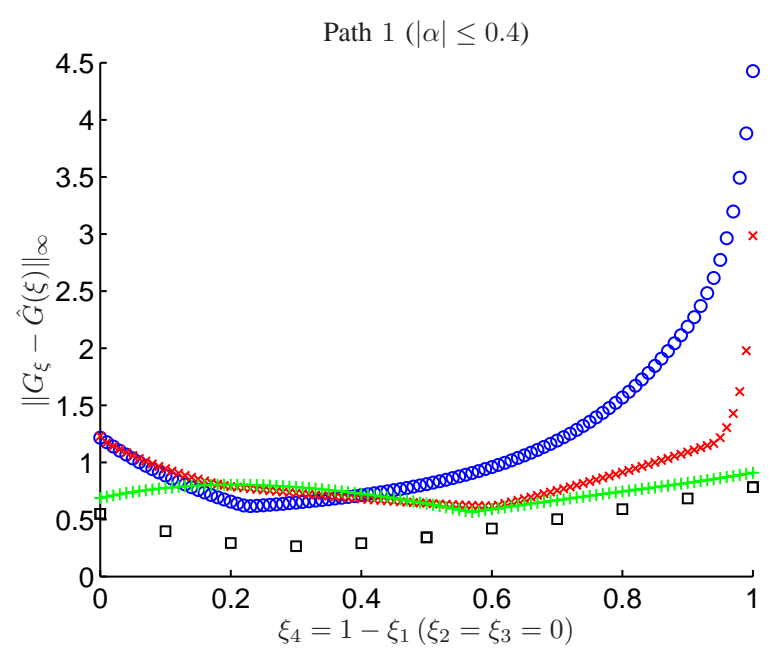

Path $2(|\alpha| \leq 0.4)$

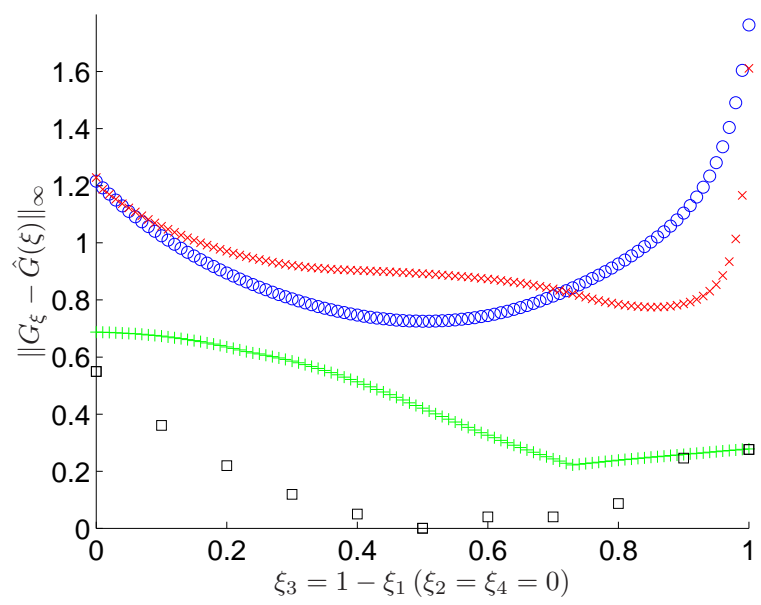

Fig. 1. Actual model approximation errors for the proposed methods applied to two parameter paths of the example: (o) corresponds to $\hat{G}_{\xi, 1},(\times)$ to $\hat{G}_{\xi, 2},(+)$ to $\hat{G}_{3}(\xi)$, and $(\square)$ corresponds to normal balanced truncation applied to some points in $M_{\xi}$ for comparison.

actually is representative of most paths in $M_{\xi}$ encountered in this study) we see that for most $\xi, \hat{G}_{3}(\xi)(+)$ is the best approximation, followed by $\hat{G}_{\xi, 2}(\times)$, and then by $\hat{G}_{\xi, 1}(\circ)$. This is the expected result from the error bound analysis and because $\hat{G}_{3}(\xi)$ has more degrees of freedom given the affine Gramians. But note that for certain $\xi$ it may happen that $\hat{G}_{\xi, 1}$ is best even if it was only rarely observed in this study. One can also note that the approximation error increases fast for $G_{\xi, 1}$ as $\xi_{4} \rightarrow 1$. An explanation is that we have chosen the region $|\alpha| \leq 0.4$, which is close to the quadratic stability limit. Thus, the LMIs (8)-(9) are close to being infeasible. The extended LMIs (12)-(13) and (16)(17) are feasible in the entire stability region $|\alpha|<0.4619$ on the other hand (deleting the constraints involving $P^{\star}$ and $\left.Q^{\star}\right)$, as shown in [11]. This provides an explanation as to why the corresponding approximations are better behaved. To summarize, the approximation $\hat{G}_{3}(\xi)$ is best, but it has a more complicated realization than $\hat{G}_{\xi, 2}$ and $\hat{G}_{\xi, 1}$.

It can also be noted that normal balanced truncation ( $\square$ in Fig. 1) is best in the points where it is applied. But 
this is expected, since normal balanced truncation tries to approximate just a single model $\left(G_{\xi}\right.$ for one $\left.\xi\right)$ and not an entire model set $\left(G_{\xi}\right.$ for all $\left.\xi \in \Xi\right)$. The reason for including these points is just to see how far off the parameter-varying techniques are from good point-wise approximations.

As can be seen in Path 1, the reduced model $\hat{G}_{3}(\xi)$ based on affine Gramians is typically not too far off the point-wise approximations. However, as seen in Path 2 at $\xi_{3}=0.5$ this is not always the case. At this point, the realization of $G_{\xi}$ is non-minimal and has McMillan degree two. Normal balanced truncation can exploit this and removes the non-minimal states, whereas the parameter-varying methods apparently cannot. The reason is that the parameter-varying balanced coordinate transformations are restricted by the chosen class of generalized Gramians. But Path 2 was an exceptional path in this study, and Path 1 is more representative among the parameter paths seen. Path 2 is nevertheless interesting since it shows a case where the introduced methods do not perform as well, and it would be interesting to study it further.

Next, the set of allowable models is shrunk by putting $|\alpha| \leq 0.04$ (a factor 10 smaller than before). The approximation errors and the error bounds from Theorem 2 are shown in Fig. 2. Two interesting aspects can be noted: First of all, the actual approximation errors of all methods improve, and become comparable. Second, the error bounds of course satisfy Theorem 2, but it is seen that it may happen for some $\xi$ that $\delta_{3}(q, \xi)>\delta_{2}(q)$.

From the example we see that with high likelihood and for randomly selected $\xi, \hat{G}_{3}(\xi)$ is the best approximation, followed by $\hat{G}_{\xi, 2}$, and then by the more traditional $\hat{G}_{\xi, 1}$. What can be said with certainty is that the error bounds are as good as, or better, for $\hat{G}_{\xi, 2}$ and $\hat{G}_{3}(\xi)$. The extended LMIs have thus proved their value also for PMOR.

\section{CONClusions}

Two new methods for solving the PMOR problem have been presented. The methods employ extended Lyapunov inequalities and their slack variables to obtain parameterized and extended Gramians through standard convex optimization techniques. These Gramians are shown to improve both actual approximation errors and error bounds.

\section{ACKNOWLEDGMENT}

The author thanks Dr. Kin Cheong Sou and the anonymous reviewers for helpful suggestions.

\section{REFERENCES}

[1] G. Obinata and B. D. O. Anderson, Model reduction for control system design, ser. Communications and control engineering. Springer, 2000.

[2] A. Antoulas, Approximation of large-scale dynamical systems, ser. Advances in design and control. Society for Industrial and Applied Mathematics, 2005.

[3] K. C. Sou, A. Megretski, and L. Daniel, "A quasi-convex optimization approach to parameterized model order reduction," Computer-Aided Design of Integrated Circuits and Systems, IEEE Transactions on, vol. 27, no. 3, pp. $456-469$, march 2008.

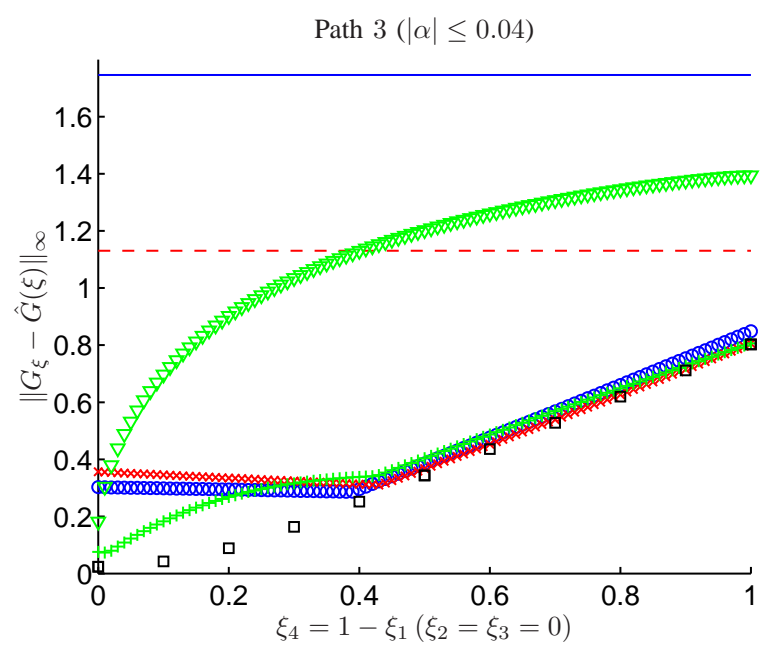

Fig. 2. Actual model approximation errors when $|\alpha| \leq 0.04$, and error bounds from Theorem 2: $\delta_{1}(2)$ is $(-), \delta_{2}(2)$ is $(--)$, and $\delta_{3}(2, \xi)$ is $(\nabla)$. Actual approximation errors denoted with same symbols as in Fig. 1. The error bound $\delta_{1}(2)$ is clearly most conservative.

[4] U. Baur, C. Beattie, P. Benner, and S. Gugercin, "Interpolatory projection methods for parameterized model reduction," SIAM J. Sci. Comput., vol. 33, pp. 2489-2518, Oct. 2011.

[5] G. Wood, P. Goddard, and K. Glover, "Approximation of linear parameter-varying systems," in Decision and Control, 1996., Proceedings of the 35th IEEE, vol. 1, dec 1996, pp. $406-411$ vol.1.

[6] C. Beck, J. Doyle, and K. Glover, "Model reduction of multidimensional and uncertain systems," Automatic Control, IEEE Transactions on, vol. 41, no. 10, pp. $1466-1477$, Oct. 1996.

[7] M. Farhood and G. E. Dullerud, "Model reduction of nonstationary LPV systems," Automatic Control, IEEE Transactions on, vol. 52, no. 2, pp. $181-196$, feb. 2007.

[8] L. Li and I. Petersen, "A Gramian-based approach to model reduction for uncertain systems," Automatic Control, IEEE Transactions on, vol. 55, no. 2, pp. $508-514$, Feb. 2010.

[9] H. Sandberg, "An extension to balanced truncation with application to structured model reduction," Automatic Control, IEEE Transactions on, vol. 55, no. 4, pp. 1038 -1043, Apr. 2010.

[10] G. Dullerud and F. Paganini, A course in robust control theory: a convex approach, ser. Texts in applied mathematics. Springer, 2000.

[11] M. de Oliveira, J. Bernussou, and J. Geromel, "A new discrete-time robust stability condition," Systems \& Control Letters, vol. 37, no. 4, pp. $261-265,1999$.

[12] M. C. De Oliveira, J. C. Geromel, and J. Bernussou, "Extended $\mathrm{H}_{2}$ and $H_{\infty}$ norm characterizations and controller parametrizations for discrete-time systems," International Journal of Control, vol. 75, no. 9 , pp. 666-679, 2002.

[13] G. Pipeleers, B. Demeulenaere, J. Swevers, and L. Vandenberghe, "Extended LMI characterizations for stability and performance of linear systems," Systems \& Control Letters, vol. 58, no. 7, pp. 510 $-518,2009$.

[14] K. Zhou, J. C. Doyle, and K. Glover, Robust and optimal control. Upper Saddle River, NJ, USA: Prentice-Hall, Inc., 1996.

[15] P. Apkarian and H. Tuan, "Parameterized LMIs in control theory," SIAM Journal on Control and Optimization, vol. 38, no. 4, pp. 12411264,2000

[16] P. A. Parrilo, "Semidefinite programming relaxations for semialgebraic problems," Mathematical Programming, vol. 96, pp. 293-320, 2003.

[17] J. F. Sturm, "Using SeDuMi 1.02, a MATLAB toolbox for optimization over symmetric cones," 1998.

[18] J. Löfberg, "YALMIP : A toolbox for modeling and optimization in MATLAB," in Proceedings of the CACSD Conference, Taipei, Taiwan, 2004. 